\title{
Estimation of Genetic Components of Cocoa Populations (Theobroma cacao L.) in Seedling Phase
}

\section{Pendugaan Nilai Komponen Genetik Populasi Kakao (Theobroma cacao L.) pada Fase Bibit}

\author{
Agung Wahyu Susilo ${ }^{1}$, Sobir $^{2 *}$, Desta Wirnas ${ }^{2}$, and Adinda Wuriandani ${ }^{3}$ \\ ${ }^{1}$ Indonesian Coffee and Cocoa Research Institute \\ Jl. PB Sudirman 90, Jember, Indonesia \\ ${ }^{2}$ Department of Agronomy and Horticulture, Faculty of Agriculture, Bogor Agricultural University \\ Jl. Meranti, Kampus IPB Dramaga, Bogor, 16680, Indonesia \\ ${ }^{3}$ Major of Plant Breeding and Biotechnology, Graduate School, Bogor Agricultural University
}

Received 23 March 2020/Accepted 24 April 2020

\begin{abstract}
Seedling is often used as planting material and rootstock for clonal propagation in cocoa. Seedling as cocoa planting material came form superior parents or crosses of superior parents. The research aimed to estimate the genetic components of cocoa morphological characters using the North Carolina II mating design. Three female parent genotypes, namely KW 516, Sulawesi 3, and TSH 858, were crossed with two male parent genotypes, namely ICCRI 09 and Scavina 6. Observed variables consisted of plant height, stem diameter, number of leaves, leaf area, root length, root volume, shoot fresh and dry weight, root fresh and dry weight, root:shoot ratio at 16 weeks after showing. General combining ability (GCA) value showed Sulawesi 3 as the best female parent, and Scavina 6 as the best male parent. Specific combining ability (SCA) values showed TSH $858 \times$ Scavina 6, KW $516 \times$ ICCRI 09, and Sulawesi 3 x ICCRI 09 as the best crosses. Sulawesi $3 \times$ ICCRI 09 seedlings had good vigor and met the criteria for transplanting. Cocoa morphological characters were affected by dominant gene action except for root volume.
\end{abstract}

Keywords: seedling, North Carolina II, general combining ability, specific combining ability

\section{ABSTRAK}

Penggunaan bibit sebagai bahan tanam maupun batang bawah untuk perbanyakan klonal sering dilakukan. Bahan tanam berupa bibit merupakan bahan tanam unggul yang berasal dari tetua unggul maupun persilangan antar tetua unggul. Penelitian ini bertujuan untuk mengetahui nilai duga komponen genetik melalui desain persilangan North Carolina II pada karakter morfologi tanaman kakao fase bibit. Persilangan dilakukan dengan menggunakan lima genotipe tetua betina, yaitu; KW 516, Sulawesi 3, TSH 858 dan dua genotipe tetua jantan, yaitu; ICCRI 09, Scavina 6. Pengamatan dilakukan pada tanaman umur 16 MST pada karakter morfologi tanaman yaitu tinggi tanaman, diameter batang, jumlah daun, panjang akar, volume akar, bobot basah tajuk, bobot basah akar, bobot kering tajuk, bobot kering akar, dan rasio akar/tajuk. Genotipe Sulawesi 3 merupakan genotipe tetua betina terbaik, sedangkan Scavina 6 merupakan genotipe tetua jantan terbaik. Genotipe persilangan terbaik berdasarkan nilai DGK nya adalah genotipe TSH 858 x Scavina 6, KW 516 x ICCRI 09, dan Sulawesi $3 x$ ICCRI 09. Genotipe persilangan Sulawesi 3 x ICCRI 09 merupakan genotipe dengan vigor yang baik yang memenuhi kriteria bibit unggul dan siap untuk ditanam di kebun. Karakter morfologi pada tanaman kakao fase bibit dikendalikan oleh gen dominan pada semua karakter kecuali pada karakter volume akar.

Kata kunci: bibit, daya gabung khusus, daya gabung umum, North Carolina II

\section{INTRODUCTION}

Cocoa is one of the leading agriculture or industrial crops in Indonesia. Indonesia is the third-largest cocoa

* corresponding author. e-mail: rsobir@yahoo.com producer in the world after Ivory Coast and Ghana (ICCO, 2017). Cocoa production in Indonesia in the last five years decreased by 32 thousand tons (Ditjenbun, 2017). According to BPS (2018), national cocoa production in 2016 reached 658399 tons, and in 2017 national cocoa production decreased by 1349 tons. 
Low productivity of cocoa can be caused by pest and disease, climate change, damaged crop canopy, decrease of population, and cultivation technology (Limbongan, 2011; Wuriandani et al., 2018). Low productivity also affected by low-quality planting materials such as old materials (Randriani and Dani, 2014). Cocoa planting material comes from a seedling. The seedling can be used as planting material or rootstock for clonal propagation. Vigorous seedling in cocoa is determined by bean position, kind of genotype, bean maturity, and environment effect (Olaiya, 2016). The seedling material comes from generative propagation, such as crossing between superior parents.

Superior planting material is the most efficient tool to overcome low productivity in cocoa. Hybrid of cocoa clones is selected based on heterosis effects in the offspring (Randriani and Dani, 2014). According to Lopes et al. (2011), one of the mating designs that can be used to develop the cocoa diversity population is North Carolina II (NCII). Each member of the male parents is crossed with each member of the female parents (Nduwumuremyi et al., 2013). NC II mating design is similar to line $\mathrm{x}$ tester mating-design because both mating-designs calculate the influence of the variance female and male parents, as well as the influence of the interaction of female $\mathrm{x}$ male parents (Fasahat et al., 2016).

The best parent for producing hybrid variety was selected by combining ability test. The best parent must have good GCA value (Randriani and Dani, 2014). Combining ability is the ability of parents if combine with other parents through crossing (Zhang et al., 2015). According to Fasahat et al. (2016), there are two types of combining ability: general combining ability (GCA) and specific combining ability (SCA). General combining ability (GCA) is the average performance value of the parent used in a cross. Specific combining ability (SCA) is the interaction effects among the female and male parents (Setyowidianto et $a l ., 2017)$. The GCA value is related to the additive gene, while the SCA value is related to non-additive gene actions (Herison et al., 2017).

The research aimed to estimate the genetic components value through the NC II mating design. Best parents were selected according to general combining ability (GCA) value, and the best combination genotypes was selected according to specific combining ability (SCA) value in the seedling phase.

\section{MATERIALS AND METHODS}

This research was conducted at Kaliwining Experimental Field, Indonesian Coffee and Cocoa Research Institute from August 2018 until May 2019. Planting activity after crossing and produced $F_{1}$ genotypes was carried out at Greenhouse Kaliwining Experimental Field, Indonesian Coffee and Cocoa Research Institute.

This research started with crossing using North Caroline II (NCII) mating-design. Female parent genotypes were TSH 858, KW 516, and Sulawesi 3, while male parent genotypes were Scavina 6 and ICCRI 09. The total number of cross combination were six combinations. Planting materials in this research consisted of six crosses (hybrids) and five parents. This research used 50 seeds with 5 replicates, each of replicate consisted of 10 seeds. Total unit of samples were $11 \times 5 \times 10=550$ unit samples.

Observations were made in plant morphological characters, such as stem diameter, root length, root volume, shoot fresh and dry weight, root fresh and dry weight, and root:shoot ratio. This observation was finished at 16 weeks after showing.

NCII mating-design flow analysis was used in this research data analysis. R 3.4 version was used as a software for analysis. Linear additive models for variance analysis was:

$$
\mathrm{Y}_{\mathrm{ijk}}=\mu+\mathrm{m}_{\mathrm{i}}+\mathrm{f}_{\mathrm{j}}+(\mathrm{m} \times \mathrm{f})_{\mathrm{ij}}+\varepsilon_{\mathrm{ijk}}
$$

$\mathrm{Y}_{\mathrm{ijk}} \quad=$ respon value on cross combination of female parent- $\mathrm{i} \times$ male parent-j, replication- $\mathrm{k}$

$\mu \quad=$ overall mean

$\mathrm{m}_{\mathrm{i}} \quad=$ effect of male parent-i

$\mathrm{f}_{\mathrm{j}} \quad=$ effect of female parent-j,

$(\mathrm{m} \times \mathrm{f})_{\mathrm{ij}}=$ interaction effect between male parent-i and female parent-j

$\varepsilon_{\mathrm{ijk}} \quad=$ experimental error

NCII mating design results consisted of GCA on the parents used, value of SCA on the cross combination produced, variance of GCA $\left(\sigma_{\mathrm{GCA}}^{2}\right)$, variance of SCA $\left(\sigma_{\mathrm{SCA}}^{2}\right)$, variance additives $\left(\sigma_{\mathrm{A}}^{2}\right)$, and variance dominant $\left(\sigma_{D}^{2}\right)$. These values then used to estimate the broad sense heritability $\left(\mathrm{h}_{\mathrm{bs}}^{2}\right)$ and narrow sense heritability $\left(\mathrm{h}^{2}{ }_{\mathrm{ns}}\right)$.

\section{RESULTS AND DISCUSSION}

Cocoa can be propagated through vegetative and generative propagation. Vegetative propagation can be done through cutting and grafting, while generative propagation can be done through seed (Sutardi \& Hendarta, 2009). Cocoa planting material such as seed can be used as superior planting material or rootstock for clonal propagation (Prawoto et al., 1990). Seedling with good quality came from superior seed and good treatment nursery (Pancaningtyas et al., 2014). Superior seed in cocoa came from crossing between superior parents having good combining ability. North Carolina II (NC II) mating design was used to develop good parent combiner and good progeny.

Analysis of variance showed all observed characters were significantly different in among genotypes. Female parents showed significant performance differences in root volume, while male parents didn't show significant differences performance in all observed characters. The interaction between female parents and male parents showed significant effect except for root volume and root:shoot ratio character (Table 1).

The results of general combined ability (GCA) analysis in morphological characters showed that Sulawesi 
Table1. Variance analysis of the morphological characters in cocoa seedling phase (16 weeks after sowing) with NCII matingdesign

\begin{tabular}{|c|c|c|c|c|c|c|c|c|c|c|c|c|}
\hline \multirow{2}{*}{ Source of variation } & \multirow{2}{*}{$\mathrm{df}$} & \multicolumn{11}{|c|}{ Mean square (MS) } \\
\hline & & $\mathrm{PH}$ & SD & NL & LA & RL & RV & SFW & RFW & SDW & RDW & $\mathrm{R} / \mathrm{S}$ \\
\hline Replication & 4 & $422.99 *$ & $0.062 *$ & $30.09 *$ & $7858.76^{*}$ & $76.87 *$ & $44.35 *$ & $377.78^{*}$ & $22.83 *$ & $35.05 *$ & $1.92 *$ & $0.0006 \mathrm{~ns}$ \\
\hline $\mathrm{F}_{1}$ & 5 & $590.06^{*}$ & $0.033^{*}$ & $14.86^{*}$ & $2794.49^{*}$ & $23.47 *$ & $11.33^{*}$ & $172.6^{*}$ & $19.07 *$ & $14.35^{*}$ & $0.41^{*}$ & $0.031 *$ \\
\hline Female parent $(\mathrm{F})$ & 2 & $829.52 \mathrm{~ns}$ & $0.010 \mathrm{~ns}$ & $4.71 \mathrm{~ns}$ & $3143.7 \mathrm{~ns}$ & $39.71 \mathrm{~ns}$ & $25.62 *$ & $230.4 \mathrm{~ns}$ & $37.41 \mathrm{~ns}$ & $12.34 \mathrm{~ns}$ & $0.67 \mathrm{~ns}$ & $0.049 \mathrm{~ns}$ \\
\hline Male parent (M) & 1 & $900.69 \mathrm{~ns}$ & $0.00003 \mathrm{~ns}$ & $26.75 \mathrm{~ns}$ & $2814.75 \mathrm{~ns}$ & $5.84 \mathrm{~ns}$ & $2.6 \mathrm{~ns}$ & $242.65 \mathrm{~ns}$ & $9.64 \mathrm{~ns}$ & $23.66 \mathrm{~ns}$ & $0.32 \mathrm{~ns}$ & $0.028 \mathrm{~ns}$ \\
\hline F X M & 2 & $195.29 *$ & $0.071 *$ & $19.07 *$ & $2435.13 *$ & $16.04 *$ & $1.41 \mathrm{~ns}$ & $79.76^{*}$ & $5.44 *$ & $11.69^{*}$ & $0.19^{*}$ & $0.016 \mathrm{~ns}$ \\
\hline Error & 40 & 5.4 & 0.005 & 0.95 & 63.65 & 0.72 & 0.45 & 3.28 & 0.73 & 0.24 & 0.032 & 0.004 \\
\hline
\end{tabular}

Note: * significant at $\alpha=5 \%$; ns not significant; $\mathrm{PH}=$ plant height; $\mathrm{SD}=$ stem diameter; $\mathrm{NL}=$ number of leaves; $\mathrm{LA}=$ leaf area; $\mathrm{RL}=$ root length; RV = root volume; SFW = shoot fresh weight; RFW = root fresh weight; SDW = shoot dry weight; RDW = root dry weight; $\mathrm{R} / \mathrm{S}=$ root : shoot ratio

3 was the best female parent because it had the best GCA values in all characters except the root length and root:shoot ratio. Scavina 6 was the best male parent because it had the best GCA value in all characters except stem diameter and root:shoot ratio (Table 2). According to Towaha and Wardiana (2015), Scavina 6 and Sulawesi 3 were moderate tolerant genotypes to drought stress. Generally, Scavina 6 clone has been used for the formation of superior hybrids in cocoa plants because Scavina 6 had tolerance in some diseases like Vascular streak dieback (VSD) (Prawoto et al., 2013). According to Zakariyya (2017), Scavina 6 genotype has long seedling root.

The result of specific combining ability (SCA) analysis in morphological characters showed TSH 858 x Scavina 6 was the best cross combination, which had good SCA value in all characters except the root:shoot ratio. KW $516 \mathrm{x}$ ICCRI 09 showed good SCA value in all characters except stem diameter and root:shoot ratio. Sulawesi 3 x ICCRI 09 also showed good SCA value in all characters except root length and root:shoot ratio (Table 3).

Positive values of GCA or SCA contribute to a higher phenotypic value of a trait, including morphological characters at the seedling phase. According to Pancaningtyas et al. (2014), criteria of good cocoa seedling are 3-5 month old in nursery, plant height is $40-60 \mathrm{~cm}$, minimum number of leaves are 12 , stem diameter is $0.7-1.0 \mathrm{~cm}$, green leaf color, minimum leaf width is $10 \mathrm{~cm}$ and minimum leaf length is $30 \mathrm{~cm}$.

The observation of morphological characters were conducted at 4 month old seedling (Table 4). Mean value for all genotype in plant height indicated good seedling performance except for KW 516 x ICCRI 09 and TSH 858 x ICCRI 09. KW 516, Scavina 6, KW 516 x Scavina 6, and Sulawesi 3 x ICCRI 09 had good stem diameter. Mean value for all genotype in the total number of leaves character indicated good seedling performance except for TSH 858

Table 2. Estimated values of general combining ability (GCA) of female dan male parents on morphological characters in cocoa seedling phase (16 weeks after sowing)

\begin{tabular}{|c|c|c|c|c|c|c|c|c|c|c|c|}
\hline \multirow{2}{*}{ Female } & \multicolumn{11}{|c|}{ GCA estimate } \\
\hline & $\mathrm{PH}$ & SD & NL & LA & RL & RV & SFW & RFW & SDW & RDW & $\mathrm{R} / \mathrm{S}$ \\
\hline KW 516 & -4.25 & 0.027 & -0.72 & -13.7 & 1.34 & -1.02 & -3.16 & -1.46 & -0.998 & -0.29 & -0.04 \\
\hline Sulawesi 3 & 10.46 & 0.008 & 0.65 & 20.03 & -2.29 & 1.85 & 5.52 & 2.2 & 1.197 & 0.075 & -0.04 \\
\hline TSH 858 & -6.21 & -0.036 & 0.08 & -6.33 & 0.95 & -0.82 & -2.36 & -0.739 & -0.199 & 0.214 & 0.081 \\
\hline SE GCA Female & 0.73 & 0.023 & 0.31 & 2.52 & 0.27 & 0.21 & 0.57 & 0.27 & 0.15 & 0.056 & 0.02 \\
\hline \multirow{2}{*}{ Male } & \multicolumn{11}{|c|}{ GCA estimate } \\
\hline & $\mathrm{PH}$ & SD & NL & LA & RL & RV & SFW & RFW & SDW & RDW & $\mathrm{R} / \mathrm{S}$ \\
\hline ICCRI 09 & -5.48 & 0.001 & -0.94 & -9.69 & -0.44 & -0.3 & -2.84 & -0.57 & -0.89 & -0.104 & 0.03 \\
\hline Scavina 6 & 5.48 & -0.001 & 0.94 & 9.69 & 0.44 & 0.3 & 2.84 & 0.57 & 0.89 & 0.104 & -0.03 \\
\hline SE GCA Male & 0.6 & 0.019 & 0.25 & 2.06 & 0.22 & 0.17 & 0.47 & 0.22 & 0.13 & 0.046 & 0.016 \\
\hline
\end{tabular}

Note: $\mathrm{PH}=$ plant height, $\mathrm{SD}=$ stem diameter, $\mathrm{NL}=$ number of leaves, $\mathrm{LA}=$ leaf area, $\mathrm{RL}=$ root length, $\mathrm{RV}=$ root volume, $\mathrm{SFW}=$ shoot fresh weight, RFW = root fresh weight, $\mathrm{SDW}=$ shoot dry weight, $\mathrm{RDW}=$ root dry weight, $\mathrm{R} / \mathrm{S}$ = root : shoot ratio, $\mathrm{SE} \mathrm{GCA}=$ standard error of general combining ability 
Table 3. Estimated value of specific combining ability (SCA) in crossing combinations on morphological characters in cocoa seedling phase (16 weeks after sowing)

\begin{tabular}{|c|c|c|c|c|c|c|c|c|c|c|c|}
\hline \multirow{2}{*}{ Genotype } & \multicolumn{11}{|c|}{ SCA estimate } \\
\hline & $\mathrm{PH}$ & SD & NL & LA & RL & RV & SFW & RFW & SDW & RDW & $\mathrm{R} / \mathrm{S}$ \\
\hline KW516xICCRI 09 & 0.83 & -0.040 & 0.68 & 10.32 & 1.46 & 0.028 & 0.50 & 0.47 & 0.21 & 0.06 & -0.01 \\
\hline KW516xSca6 & -0.83 & 0.040 & -0.68 & -10.32 & -1.46 & -0.028 & -0.50 & -0.47 & -0.21 & -0.06 & 0.01 \\
\hline Sul3xICCRI 09 & 3.95 & 0.097 & 0.91 & 7.63 & -0.65 & 0.361 & 2.54 & 0.38 & 0.96 & 0.09 & -0.03 \\
\hline Sul3xSca6 & -3.95 & -0.097 & -0.91 & -7.63 & 0.65 & -0.361 & -2.54 & -0.38 & -0.96 & -0.09 & 0.03 \\
\hline TSH858xICCRI 09 & -4.77 & -0.057 & -1.59 & -17.95 & -0.81 & -0.39 & -3.04 & -0.85 & -1.17 & -0.16 & 0.05 \\
\hline TSH858xSca6 & 4.77 & 0.057 & 1.59 & 17.95 & 0.81 & 0.39 & 3.04 & 0.85 & 1.17 & 0.16 & -0.05 \\
\hline SE SCA & 1.04 & 0.032 & 0.44 & 3.57 & 0.38 & 0.30 & 0.81 & 0.38 & 0.22 & 0.08 & 0.03 \\
\hline
\end{tabular}

Note: $\mathrm{PH}=$ plant height; $\mathrm{SD}=$ stem diameter; $\mathrm{NL}=$ number of leaves; $\mathrm{LA}=$ leaf area; $\mathrm{RL}=$ root length; $\mathrm{RV}=$ root volume; $\mathrm{SFW}=$ shoot fresh weight; RFW = root fresh weight; SDW = shoot dry weight; RDW = root dry weight; R/S = root : shoot ratio; SE SCA = standard error of specific combining ability

Table 4. Mean values of the observed characters in five parents and six F1 hybrids of cocoa in seedling phase (16 weeks after showing)

\begin{tabular}{|c|c|c|c|c|c|c|c|c|c|c|c|}
\hline \multirow[b]{2}{*}{ Genotype } & \multicolumn{11}{|c|}{ Mean value } \\
\hline & $\begin{array}{l}\mathrm{PH} \\
(\mathrm{cm})\end{array}$ & $\begin{array}{l}\text { SD } \\
(\mathrm{cm})\end{array}$ & NL & $\begin{array}{c}\text { LA } \\
\left(\mathrm{cm}^{2}\right)\end{array}$ & $\begin{array}{l}\mathrm{RL} \\
(\mathrm{cm})\end{array}$ & $\begin{array}{c}\mathrm{RV} \\
(\mathrm{mL})\end{array}$ & $\begin{array}{c}\text { SFW } \\
\text { (g) }\end{array}$ & $\begin{array}{c}\text { RFW } \\
\text { (g) }\end{array}$ & $\begin{array}{l}\text { SDW } \\
(\mathrm{g})\end{array}$ & $\begin{array}{l}\text { RDW } \\
(\mathrm{g})\end{array}$ & $\mathrm{R} / \mathrm{S}$ \\
\hline \multicolumn{12}{|l|}{ Parent } \\
\hline KW 516 & $53.02 b c$ & $0.76 \mathrm{a}$ & $15.7 \mathrm{~cd}$ & $122.63 b$ & $18.81 \mathrm{~cd}$ & $6.20 \mathrm{ab}$ & $24.07 \mathrm{bc}$ & $5.84 \mathrm{ab}$ & $7.05 \mathrm{~b}$ & $1.93 \mathrm{a}$ & $0.28 \mathrm{bc}$ \\
\hline Sulawesi 3 & $49.51 \mathrm{de}$ & $0.65 b-d$ & $15.6 \mathrm{~cd}$ & $141.75 \mathrm{a}$ & $16.58 \mathrm{f}$ & $5.90 \mathrm{a}-\mathrm{c}$ & $24.30 \mathrm{~b}$ & $6.67 \mathrm{ab}$ & $6.67 b$ & $1.11 \mathrm{~cd}$ & $0.16 \mathrm{~d}$ \\
\hline TSH 858 & $41.11 \mathrm{f}$ & $0.57 \mathrm{de}$ & $13.0 \mathrm{e}$ & $98.49 \mathrm{c}$ & $17.62 \mathrm{~d}-\mathrm{f}$ & $2.60 \mathrm{e}$ & $12.08 \mathrm{~g}$ & $3.07 \mathrm{~d}$ & $3.54 \mathrm{ef}$ & $1.14 \mathrm{~cd}$ & $0.39 \mathrm{a}$ \\
\hline ICCRI 09 & $56.98 \mathrm{a}$ & $0.67 a-d$ & $17.5 \mathrm{a}$ & $141.47 \mathrm{a}$ & $19.40 \mathrm{bc}$ & $6.80 \mathrm{a}$ & $28.97 \mathrm{a}$ & $6.90 \mathrm{a}$ & $8.05 \mathrm{a}$ & $1.41 \mathrm{~b}$ & $0.17 \mathrm{~d}$ \\
\hline Scavina 6 & $53.43 \mathrm{bc}$ & $0.74 \mathrm{ab}$ & $16.4 \mathrm{bc}$ & $124.28 b$ & $20.60 \mathrm{a}$ & $5.23 \mathrm{c}$ & $22.40 \mathrm{~b}-\mathrm{d}$ & $5.70 \mathrm{~b}$ & $5.54 \mathrm{~d}$ & $0.97 \mathrm{de}$ & $0.17 \mathrm{~d}$ \\
\hline \multicolumn{12}{|l|}{$\mathrm{F}_{1}$} \\
\hline KW516xICCRI 09 & $34.15 \mathrm{~g}$ & $0.62 \mathrm{c}-\mathrm{e}$ & $13.2 \mathrm{e}$ & $91.22 \mathrm{c}$ & $20.74 \mathrm{a}$ & $2.67 \mathrm{e}$ & $10.33 \mathrm{~g}$ & $2.20 \mathrm{de}$ & $2.84 \mathrm{fg}$ & $0.55 \mathrm{~g}$ & $0.18 \mathrm{~d}$ \\
\hline KW516xSca6 & $43.42 \mathrm{f}$ & $0.7 \mathrm{a}-\mathrm{c}$ & $13.7 \mathrm{e}$ & $89.95 \mathrm{c}$ & $18.70 \mathrm{~cd}$ & $3.20 \mathrm{de}$ & $15.03 \mathrm{f}$ & $2.40 \mathrm{de}$ & $4.19 \mathrm{e}$ & $0.63 \mathrm{fg}$ & $0.14 \mathrm{~d}$ \\
\hline Sul3xICCRI 09 & $51.98 \mathrm{~cd}$ & $0.74 \mathrm{ab}$ & $14.8 \mathrm{~d}$ & $122.25 b$ & $15.00 \mathrm{~g}$ & $5.87 \mathrm{a}-\mathrm{c}$ & $21.07 \mathrm{de}$ & $5.77 \mathrm{ab}$ & $5.78 \mathrm{~cd}$ & $0.94 \mathrm{de}$ & $0.16 \mathrm{~d}$ \\
\hline Sul3xSca6 & $55.04 \mathrm{ab}$ & $0.54 \mathrm{e}$ & $14.9 \mathrm{~d}$ & $126.37 b$ & $17.18 \mathrm{ef}$ & $5.73 b c$ & $21.67 \mathrm{c}-\mathrm{e}$ & $6.13 \mathrm{ab}$ & $5.64 d$ & $0.96 \mathrm{de}$ & $0.16 \mathrm{~d}$ \\
\hline
\end{tabular}

Note: Numbers followed by the same letter in the same column are not significantly different based on the DMRT at $\alpha=5 \%$. PH $=$ plant height; $\mathrm{SD}=$ stem diameter; $\mathrm{NL}=$ number of leaves; $\mathrm{LA}=$ leaf area; $\mathrm{RL}=$ root length; $\mathrm{RV}=$ root volume; $\mathrm{SFW}=$ shoot fresh weight; $\mathrm{RFW}=$ root fresh weight; SDW = shoot dry weight; RDW = root dry weight; R/S = root : shoot ratio

x ICCRI 09. These results showed KW 516, Scavina 6, KW 516 x Scavina 6, and Sulawesi 3 x ICCRI 09 as good seedling genotypes.

The effect of gene action on a plant character can be seen through the ratio of GCA and SCA variances. According to Istipliler et al. (2015), smaller value of ratio degree $\sigma_{\mathrm{GCA}}^{2} / \sigma_{\mathrm{SCA}}^{2}$ than the value of dominance ratio degree $\left(\sigma_{D}^{2} / \sigma_{A}^{2}\right)^{1 / 2}$ indicated dominant gene action on the character. Genetic component values showed that all characters were influenced by dominant gene except root volume. On the other hand, root volume was influenced by additive gene action. Broad sense heritability $\left(\mathrm{h}^{2}{ }_{\mathrm{bs}}\right)$ estimates were high in all characters except root:shoot ratio (Table 5). The broad sense heritability can be classified as high $(\mathrm{H}>50 \%)$, moderate $(20 \%<\mathrm{H}<50 \%)$, and low $(\mathrm{H}<20 \%)$ (Seker et al., 2014).

The contribution proportion shows how much contribution of female parents, male parents, and their interactions for each character (Istipliler et al., 2015). The results of the analysis found that female parents strongly influenced all characters except stem diameter and number of leaves. Interaction between female and male parents had a high contribution on these two characters (Table 6). 
Table 5. Estimated genetic components value in morphological character of cocoa in seedling phase (16 weeks after sowing)

\begin{tabular}{|c|c|c|c|c|c|c|c|c|c|c|c|}
\hline \multirow{2}{*}{ Genetic component } & \multicolumn{11}{|c|}{ Estimate } \\
\hline & $\mathrm{PH}$ & SD & NL & LA & RL & RV & SFW & RFW & SDW & RDW & $\mathrm{R} / \mathrm{S}$ \\
\hline$\sigma_{\mathrm{GCA}}^{2}$ & 18.80 & 0.00 & 0.00 & 17.11 & 0.35 & 0.47 & 4.42 & 0.650 & 0.130 & 0.010 & 0.001 \\
\hline$\sigma_{\mathrm{SCA}}^{2}$ & 37.98 & 0.01 & 3.62 & 474.30 & 3.06 & 0.19 & 15.30 & 0.942 & 2.290 & 0.032 & 0.002 \\
\hline$\sigma_{\mathrm{A}}^{2}$ & 37.60 & 0.00 & 0.00 & 34.22 & 0.71 & 0.95 & 8.84 & 1.300 & 0.250 & 0.020 & 0.002 \\
\hline$\sigma_{D}^{2}$ & 37.98 & 0.01 & 3.62 & 474.30 & 3.06 & 0.19 & 15.30 & 0.940 & 2.290 & 0.030 & 0.002 \\
\hline$\sigma_{\mathrm{GCA}}^{2} / \sigma_{\mathrm{SCA}}^{2}$ & 0.50 & 0.00 & 0.00 & 0.04 & 0.11 & 2.45 & 0.29 & 0.690 & 0.057 & 0.317 & 0.292 \\
\hline$\left(\sigma_{\mathrm{GCA}}^{2} / \sigma_{\mathrm{SCA}}^{2}\right)^{1 / 2}$ & 1.01 & 0.00 & 0.00 & 3.72 & 2.08 & 0.45 & 1.32 & 0.850 & 3.027 & 1.225 & 1.265 \\
\hline $\mathrm{h}_{\mathrm{ns}}^{2}$ & 0.46 & 0.00 & 0.00 & 0.06 & 0.16 & 0.60 & 0.32 & 0.438 & 0.090 & 0.244 & 0.190 \\
\hline $\mathrm{h}^{2}{ }_{\mathrm{bs}}$ & 0.93 & 0.72 & 0.79 & 0.89 & 0.84 & 0.72 & 0.88 & 0.754 & 0.914 & 0.610 & 0.494 \\
\hline
\end{tabular}

Note: $\sigma_{\mathrm{GCA}}^{2}=$ general combining ability $(\mathrm{GCA})$ variance, $\sigma_{\mathrm{SCA}}^{2}=$ specific combining ability (SCA) variance, $\sigma_{\mathrm{A}}^{2}=$ additive variance, $\sigma^{2}$ $=$ dominant variance, $\sigma_{\mathrm{GCA}}^{2} / \sigma_{\mathrm{SCA}}^{2}=\mathrm{GCA}$ variance/SCA variance ratio, $\left(\sigma_{\mathrm{GCA}}^{2} / \sigma_{\mathrm{SCA}}^{2}\right)^{1 / 2}=$ dominance ratio degree, h2ns $=$ narrow sense heritability, $\mathrm{h}^{2}{ }_{\mathrm{bs}}=$ broad sense heritability. $\mathrm{PH}=$ plant height $(\mathrm{cm}) ; \mathrm{SD}=$ stem diameter $(\mathrm{cm}) ; \mathrm{NL}=$ number of leaves; $\mathrm{LA}=$ leaf area $\left(\mathrm{cm}^{2}\right) ; \mathrm{RL}=$ root length $(\mathrm{cm}) ; \mathrm{RV}=$ root volume $(\mathrm{mL}) ; \mathrm{SFW}=$ shoot fresh weight $(\mathrm{g}) ; \mathrm{RFW}=$ root fresh weight $(\mathrm{g})$; SDW $=$ shoot dry weight $(\mathrm{g}) ; \mathrm{RDW}=$ root dry weight $(\mathrm{g}) ; \mathrm{R} / \mathrm{S}=$ root $:$ shoot ratio

Table 6. The contribution proportion (\%) of the female parent, male parent, and female $\mathrm{x}$ male parent interaction on the hybrid

\begin{tabular}{lrrrrrrrrrrc}
\hline \multirow{2}{*}{ Source } & \multicolumn{10}{c}{ Contribution proportion (\%) } \\
\cline { 2 - 13 } & PH & \multicolumn{1}{c|}{ SD } & \multicolumn{1}{c}{ NL } & \multicolumn{1}{c}{ LA } & \multicolumn{1}{c}{ RL } & \multicolumn{1}{c}{ RV } & SFW & RFW & SDW & RDW & R/S \\
\hline Female (F) & 56.23 & 12.77 & 12.68 & 44.99 & 67.68 & 90.43 & 53.40 & 78.48 & 34.42 & 65.78 & 62.12 \\
Male (M) & 30.53 & 0.02 & 36.01 & 20.15 & 4.98 & 4.60 & 28.12 & 10.12 & 32.98 & 15.89 & 17.57 \\
F x M & 13.24 & 87.21 & 51.32 & 34.86 & 27.34 & 4.97 & 18.49 & 11.40 & 32.61 & 18.34 & 20.32 \\
\hline
\end{tabular}

Note: $\mathrm{PH}=$ plant height $(\mathrm{cm}) ; \mathrm{SD}=$ stem diameter $(\mathrm{cm}) ; \mathrm{NL}=$ number of leaves; $\mathrm{LA}=$ leaf area $\left(\mathrm{cm}^{2}\right) ; \mathrm{RL}=$ root length $(\mathrm{cm}) ; \mathrm{RV}=$ root volume $(\mathrm{mL}) ; \mathrm{SFW}=$ shoot fresh weight $(\mathrm{g}) ; \mathrm{RFW}=$ root fresh weight $(\mathrm{g}) ; \mathrm{SDW}=$ shoot dry weight $(\mathrm{g}) ; \mathrm{RDW}=$ root dry weight $(\mathrm{g}) ; \mathrm{R} / \mathrm{S}=$ root $:$ shoot ratio

\section{CONCLUSION}

Relatively high general combining ability (GCA) values in almost all characters were showed in Sulawesi 3 as female parent and Scavina 6 as male parent. Both parents could be selected as good female and male combiner. Relatively high specific combining ability (SCA) values in almost all characters were showed in TSH 858 x Scavina 6, KW 516 x ICCRI 09, and Sulawesi 3 x ICCRI 09, making those to be considered as the best crossing combinations. Sulawesi 3 x ICCRI 09 fulfilled the criteria of superior seedling or planting materials. Morphological characters in this research, except root volume were influenced by dominant gene action.

\section{REFERENCE}

[BPS] Badan Pusat Statistik. 2018. Statistik Kakao Indonesia 2017. Badan Pusat Statistik. Jakarta, ID.
[Ditjenbun] Direktorat Jenderal Perkebunan. 2017. Statistik Perkebunana Indonesia 2015-2017, Kakao. Direktorat Jenderal Perkebunan. Jakarta, ID.

Fasahat, P., A. Rajabi, M.J. Rad, J. Derera. 2016. Principles and utilization of combining ability in plant breeding. Biom. Biostat. Int. J. 4:1-24.

Herison, C., Rustikawati, M. Handajaningsih. 2017. Daya gabung dan heterobeltiosis karakter pertumbuhan dan hasil beberapa galur backcross cabai merah toleran CMV pada kondisi terinokulasi. J. Agron. Indonesia 45:292-298.

[ICCO] International Cocoa Organization. 2017. Production of cocoa beans. ICCO Quarterly Bulletin of Cocoa Statistic, Abidjan, CTI. 
Istipliler, D., E. Ilker, F.A. Tonk, G. Civi, M. Tosun. 2015. Line $\mathrm{x}$ tester analysis and estimating combining abilities for yield and some yield components in bread wheat. Turk. J. Field Crops. 20:72-77.

Limbongan, J. 2011. Kesiapan penerapan teknologi sambung samping untuk mendukung program rehabilitasi tanaman kakao. JPPP. 30:156-163.

Lopes, U.V., W.R. Monteiro, J.L. Pires, D. Clement, M.M. Yamada, K.P. Gramacho. 2011. Cacao breeding in Bahia, Brazil-strategies and result. Crop Breed. Appl. Biotechnol. 73-81.

Nduwumuremyi, A., P. Tongoona, S. Habimana. 2013. Mating design: helpful tool for quantitative plant breeding analysis. J. Plant Breed. Genet. 1:117-129.

Olaiya, A.O. 2016. Growth and development of cacao (Theobroma cacao L.) seedlings in the nursery as influenced by pod maturity and retaining period after harvesting. Nigerian J. Ecology. 15:19-23.

Pancaningtyas, S., T.I. Santoso, Sudarsianto. 2014. Studi perkecambahan benih kakao melalui metode perendaman. Pelita Perkeb. 30:190-197.

Prawoto, A.A., W. Soerodikoesoemo, S. Sastriowinoto, H. Hartiko. 1990. Kajian okulasi pada tanaman kakao (Theobroma cacao L.) V. Pengaruh batang bawah terhadap daya hasil batas atas. Pelita Perkeb. 6:1320.

Prawoto, A.A., T.I. Santoso, Marifah, L. Hartanto, Sutikno. 2013. Terpene profile, leaf anatomy, and enzyme activity of resistant and susceptible cocoa clones to vascular streak dieback disease. Pelita Perkeb. 29:197-209.
Randriani, E., Dani. 2014. Perakitan varietas unggul kakao untuk meningkatkan potensi daya hasil dan ketahanan terhadap. Bunga Rampai. 2014:29-38.

Seker, H., A. Yazici, P. Uysal. 2014. Analysis of variability, and genetic advance in seed yield and related traits of orchardgrass (Dactylis glomerate L.) populations. Turk. J. Agric. For. 38:633-643.

Setyowidianto, E.P., N. Basuki, Damanhuri. 2017. Daya gabung dan heterosis galur jagung (Zea mays L.) pada karakter hasil dan komponen hasil. J. Agron. Indonesia. 45:124-129.

Sutardi, R. Hendrata. 2009. Respon bibit kakao pada bagian pangkal, tengah dan pucuk terhadap pemupukan majemuk. Agrovigor. 2:103-109.

Towaha, J., E. Wardiana. 2015. Evaluasi tingkat toleransi 35 genotipe kakao terhadap periode kering. J. TIDP. 2:133-142.

Wuriandani, A., A.W. Susilo, S. Mitrowiardjo, B. Setyawan, I.A. Sari. 2018. Diversity of pods and beans of twelve cocoa clones (Theobroma cacao L.) in rainy and dry seasons. Pelita Perkeb. 34:1-10.

Zakariyya, F. 2017. Karakter morfologi perakaran beberapa semaian klon kakao asal biji. Seminar, Ekspo dan Diskusi (SEEDs) Perbenihan Nasional. Jember 27 November 2017.

Zhang, X., L. Lv, C. Lv, B. Guo, R. Xu. 2015. Combining ability of different agronomic traits and yield components in hybrid barley. Plos One. 2015:1-9. 